\title{
THE TRACE IN FINITE OPERATOR ALGEBRAS
}

\author{
RICHARD V. KADISON ${ }^{1}$
}

1. Introduction. In [3] we gave a short proof of the additivity of the trace in a factor of type $\mathrm{II}_{1}$. In the present note, we supply the comments necessary to extend this proof to von Neumann algebras of type $\mathrm{II}_{1}$. J. Dixmier proves this in [2, Chapter III, §8] with the aid of his "Théorème d'approximation" ( $a$ result of independent interest and importance in the subject; cf. [2, Chapter III, $\$ 5$ and $\S 8])$. He uses it in passing from his approximate trace which is local to a global trace. For a factor, "local" is the same as "global"; and the passage from an approximate trace to a global trace can be easily effected in [3] by reference to the $w^{*}$-compactness of the state space. In $\S 2$, we define a "center state." The systematic use of this concept yields a global approximate trace. An easy extension of Alaoglu's result [1] establishes the compactness of the center states in an appropriate topology. We have put this extension in a general form which seems to unify the treatment of the various standard variants of Alaoglu's result. In $\S 3$, we list those modifications of the proof in [3] which make it valid for von Neumann algebras of type $\mathrm{II}_{1}$.

2. A weak-compactness principle. Let $V$ and $V^{\prime}$ be real or complex linear spaces and $F$ a separating family of linear functionals on $V^{\prime}$. Denote by $t^{\prime}$ the weak- $F$ topology on $V^{\prime}$; and let $S$ be a subset of $V$ which spans it linearly, $S^{\prime}$ a $t^{\prime}$-compact subset of $V^{\prime}, s$ the set of linear transformations of $V$ into $V^{\prime}$ which map $S$ into $S^{\prime}$, and $t$ the point-open topology on the space of linear transformations of $V$ into $V^{\prime}$, where $V^{\prime}$ is taken in the $t^{\prime}$ topology (i.e. a subbase for the open sets of $t$ is the family of sets consisting of all linear transformations which map a given point in $V$ into a given $t^{\prime}$-open set in $V^{\prime}$ ).

THEOREM. $S$ is $t$-compact.

Proof. Each $T$ in $S$ corresponds to a point $T^{\prime}$ in $X$, the product of copies of $S^{\prime}$ indexed by points of $S$, defined by $T^{\prime}(x)=T x$, for each $x$ in $S$. The mapping, $T \rightarrow T^{\prime}$, is one-one and a homeomorphism of $S$ in the $t$-topology with its image, $s^{\prime}$, in the topology induced by the product topology on $X$ (each copy of $S^{\prime}$ taken in the $t^{\prime}$ topology).

Received by the editors October 24, 1960.

1 Alfred P. Sloan Fellow. 
Since $X$ is compact in this topology, it remains to show that $S^{\prime}$ is closed in $X$.

Let $p$ be a limit point of $\mathcal{S}^{\prime}$ in $X$, and let $p(x)$ be its coordinate in $S^{\prime}$ corresponding to $x$ in $S$. Then $p$ is a mapping of $S$ into $S^{\prime}$. Let $R$ be its (possibly many-valued) linear extension to $V$ (recall that $S$ spans $V$ ). It suffices to prove that $\alpha_{1} p\left(x_{1}\right)+\cdots+\alpha_{n} p\left(x_{n}\right)=0$ if $\alpha_{1} x_{1}+\cdots$ $+\alpha_{n} x_{n}=0$, with $x_{1}, \cdots, x_{n}$ in $S$, in order to show that $R$ is singlevalued and linear. Let $f$ in $F$ and $\epsilon>0$ be given. With $c=1+\left|\alpha_{1}\right|$ $+\cdots+\left|\alpha_{n}\right|$, choose $T$ in $\delta$ such that $\left|f\left[T^{\prime}\left(x_{j}\right)-p\left(x_{j}\right)\right]\right|<\epsilon / c$. Then

$\left|f\left[\alpha_{1} p\left(x_{1}\right)+\cdots+\alpha_{n} p\left(x_{n}\right)\right]\right|$

$=\left|f\left[\alpha_{1} T^{\prime}\left(x_{1}\right)+\cdots+\alpha_{n} T^{\prime}\left(x_{n}\right)-\alpha_{1} p\left(x_{1}\right)-\cdots-\alpha_{n} p\left(x_{n}\right)\right]\right|<\epsilon$.

Since $F$ is separating for $V^{\prime}$ and $\epsilon$ is an arbitrary positive number, $\alpha_{1} p\left(x_{1}\right)+\cdots+\alpha_{n} p\left(x_{n}\right)=0$. Clearly, $p=R^{\prime} \in \mathcal{S}^{\prime}$.

Corollary. Let $\left(S_{\alpha}\right)$ be a family of subsets of $S,\left(S_{\alpha}^{\prime}\right)$ a family of $t^{\prime}$-compact subsets of $S^{\prime}$, and $S_{0}$ the subset of $S$ consisting of those transformations which map $S_{\alpha}$ into $S_{\alpha}^{\prime}$, for each $\alpha$. Then $S_{0}$ is $t$-compact.

PROOF. Let $S_{\alpha}$ be the subset of $\delta$ consisting of those transformations which map $S_{\alpha}$ into $S_{\alpha}^{\prime}$. Then $\delta_{0}=\bigcap_{\alpha} \delta_{\alpha}$. Let $\delta_{y}$ be the subset of $\delta$ consisting of those transformations which map a given point, $y$, in $S_{\alpha}$ into $S_{\alpha}^{\prime}$. Then $\delta_{\alpha}=\bigcap_{y} \delta_{y}$. Finally, each $\delta_{y}$ is $t$-closed, by definition of the point-open topology.

Some special instances follow:

1. $V$ and $V^{\prime}$ are normed spaces, with $V^{\prime}$ reflexive, $S$ and $S^{\prime}$ are their unit balls, and $F$ is the dual of $V^{\prime}$.

2. With $V^{\prime}$, the scalars and the rest as in 1 gives Alaoglu's result.

3. With $V$ and $V^{\prime}$ the same Hilbert space and the rest as in 1 , yields the compactness of the unit ball in all bounded operators provided with the weak-operator topology, and from this, the compactness of the unit ball in a von Neumann algebra.

4. From 3, with $V^{\prime}$ a von Neumann algebra, $S^{\prime}$ its unit ball, $F$ all functionals of the form $T \rightarrow(T x, y)$, with $x$ and $y$ vectors in the underlying Hilbert space-in particular, with $V$ a von Neumann algebra and $S$ its unit ball-we have the compactness of the unit ball in the space of continuous linear mappings of $V$ into $V^{\prime}$. We call the $t$ topology on this space of mappings the weak-operator topology.

5. With $V, V^{\prime}$, and $F$, as in $4, S, S^{\prime}$ the positive operators in the unit spheres of $V$ and $V^{\prime}$, and $S_{1}=S_{1}^{\prime}=(I)$, we have the weak-operator compactness of the set of positive normalized linear mappings of 
$V$ into $V^{\prime}$. With $V^{\prime}$ the scalars, we have the compactness of the state space.

6. Our application will be to the situation in 5 , where $V^{\prime}$ is the center of $V, S_{\alpha}=S_{\alpha}^{\prime}$ ranging through the single-point sets of the center. The resulting positive linear mappings into the center which are the identity on the center will be called "center states."

If $r$ is a center state of $\mathbb{R}$ with center $\mathcal{C}$, then $r(C T)=\operatorname{Cr}(T)$, for each $C$ in $\mathcal{C}$ and $T$ in $R$, as follows from the first statement of $[5$, Lemma 2]. However, both follow from:

LEMma. If $r$ is a positive linear mapping of one $C^{*}$ algebra, $\mathfrak{A}$, into another, $\mathfrak{B}$, which maps an abelian self-adjoint subalgebra, $a$, of $\mathfrak{A}$ homomorphically into the center of $\mathfrak{B}$ and I onto $I$; then $r(C A)=r(C) r(A)$, for each $C$ in $a$ and each $A$ in $\mathfrak{A}$.

Proof. With $p$ a pure state of $\mathfrak{B}, p$ is multiplicative on the center of $\mathfrak{B}$, so that $\operatorname{pr}\left([C-\operatorname{pr}(C) I]^{2}\right)=0$, for each $C$ in $a$; and $C-\operatorname{pr}(C) I$ is in the left kernel of $\operatorname{pr}$. Thus, $0=\operatorname{pr}[C A-\operatorname{pr}(C) A]=\operatorname{pr}(C A)$ $-\operatorname{pr}(C) \operatorname{pr}(A)$. With $\mathfrak{B}$ the scalars, $r$ is a state of $\mathfrak{A}$ which is multiplicative (i.e. pure) on $a$; and, from the above, $r(C A)=r(C) r(A)$. Applying this to $p$ on $\mathfrak{B}$, we have $\operatorname{pr}(C) \operatorname{pr}(A)=p[r(C) r(A)]$; whence $r(C A)$ $=r(C) r(A)$, in the general case.

In the above proof, we need only that $r$ maps $Q$ into an abelian selfadjoint subalgebra of $\mathfrak{B}$ with the property that the set of state extensions of its pure states separate $\mathfrak{B}$. However:

COROLlARY. The set of states of $\mathfrak{A}$ which restrict to pure states of the abelian self-adjoint subalgebra $\mathfrak{H}_{0}$ of $\mathfrak{A}$ separates $\mathfrak{A}$ if and only if $\mathfrak{A}_{0}$ is contained in the center of $\mathfrak{A}$.

Proof. Since each pure state of $\mathfrak{A}$ is multiplicative on the center, it is pure on each subalgebra of the center. If $p$ restricts to a pure state of $\mathfrak{A}_{0}$, then, from the above lemma, $p(A C-C A)=0$, for each $A$ in $\mathfrak{A}$ and $C$ in $\mathfrak{A}_{0}$. By hypothesis, then, $A C=C A$.

3. The trace. Modify the proof of $[3, \S 2]$ as follows, to get one valid for von Neumann algebras of type $\mathrm{II}_{1}$. Read "center state" for "state," "positive multiple of a center state" for "positive linear functional," "weak-operator compactness" for "weak compactness," "projection with scalar dimension" for "nonzero projection," "von Neumann algebra of type $\mathrm{II}_{1}$ " for "factor of type $\mathrm{II}_{1}$." As dimension function, use the one constructed in $[4, \S 3.2]$. To construct $\eta$ (i.e., a normal center state), in the last paragraph of [3], let $R$ be an abelian projection with central carrier $I$ in the commutant, $\mathfrak{e}^{\prime}$, of the center, 
$\mathfrak{e}$, of $M$. Then $R \mathbb{e}^{\prime} R=\mathfrak{e} R$, and the representation $C R$ of an operator in $\mathfrak{e} R$ is unique, since $R$ has central carrier $I$. Let $\eta(T)=C$, where $R T R=C R$, for $T$ in $M$.

AdDed IN PRoof. The last paragraph of [3] should be replaced by the following for the present proof. All projections mentioned lie in $M$ and are nonzero. If each subprojection of $G$ contains a subprojection $F$ such that $\eta(F) D(G)<D(F) \eta(G)$, then a maximal orthogonal family $\left\{G_{\alpha}\right\}$ of subprojections of $G$ such that $\eta\left(G_{\alpha}\right) D(G)$ $<D\left(G_{\alpha}\right) \eta(G)$ has sum $G$. Since $\eta$ is normal, $\sum_{\alpha} \eta\left(G_{\alpha}\right) D(G)$ $=\eta\left(\sum G_{\alpha}\right) D(G)=\eta(G) D(G)<\sum D\left(G_{\alpha}\right) \eta(G)=D(G) \eta(G)$. Thus $G$ has a subprojection $F$ each subprojection $F^{\prime}$ of which satisfies $\eta\left(F^{\prime}\right) D(G)$ $\Varangle D\left(F^{\prime}\right) \eta(G)$. If $D\left(F^{\prime}\right) \eta(G) \square \eta\left(F^{\prime}\right) D(G)$, then for some central projection $Q, Q \eta\left(F^{\prime}\right) D(G)=\eta\left(F^{\prime} Q\right) D(G)<D\left(F^{\prime} Q\right) \eta(G)$. But $F^{\prime} Q$ is a subprojection of $F$ contradicting the property of $F$. Thus $D\left(F^{\prime}\right) \eta(G)$ $\leqq \eta\left(F^{\prime}\right) D(G)$ for each $F^{\prime} \leqq F$. The same argument with each inequality sign reversed shows that $G$ has a subprojection $H$ such that each $H^{\prime} \leqq H$ satisfies $D\left(H^{\prime}\right) \eta(G) \geqq \eta\left(H^{\prime}\right) D(G)$. Take $I$ for $G$, so that $D\left(F^{\prime}\right) \leqq \eta\left(F^{\prime}\right)$ when $F^{\prime} \leqq F$. Let $a=\inf \left\{\sup \left\{b: b D\left(F^{\prime}\right) \leqq \eta\left(F^{\prime}\right)\right\}: F^{\prime} \leqq F\right\}$. Then $a \geqq 1$, by choice of $F$, and $a D\left(E^{\prime}\right) \leqq \eta\left(E^{\prime}\right)$, for each $E^{\prime} \leqq F$. Choose $F^{\prime} \leqq F$, such that $c D\left(F^{\prime}\right) \$ \eta\left(F^{\prime}\right)$, where $c=(n+1) a / n$. For a suitably chosen central projection $Q, \eta\left(F^{\prime} Q\right)<c D\left(F^{\prime} Q\right)$. We may assume that $\eta\left(F^{\prime}\right)<c D\left(F^{\prime}\right)$. Next, choose $E \leqq F^{\prime}$ such that $D\left(E^{\prime}\right) \eta\left(F^{\prime}\right)$ $\geqq \eta\left(E^{\prime}\right) D\left(F^{\prime}\right)$, when $E^{\prime} \leqq E$. Then $\eta\left(E^{\prime}\right) D\left(F^{\prime}\right) \leqq c D\left(E^{\prime}\right) D\left(F^{\prime}\right)$. Since $E^{\prime} \leqq F^{\prime} \leqq F, a D\left(E^{\prime}\right) \leqq \eta\left(E^{\prime}\right)$, and $\eta\left(E^{\prime}\right) \leqq \eta\left(F^{\prime}\right)<c D\left(F^{\prime}\right)$. We may, therefore, write $\eta\left(E^{\prime}\right) \leqq c D\left(E^{\prime}\right)$ in place of $\eta\left(E^{\prime}\right) D\left(F^{\prime}\right) \leqq c D\left(E^{\prime}\right) D\left(F^{\prime}\right)$. Thus $a D\left(E^{\prime}\right) \leqq \eta\left(E^{\prime}\right) \leqq c D\left(E^{\prime}\right)$.

Using $\eta / a$ for $\rho$, from the first part of the proof in [3], there is a center state $\psi_{n}$ of $M Q$ such that $\psi_{n}\left(B^{*} B\right) \leqq(n+1) \psi_{n}\left(B B^{*}\right) / n$, for each $B$ in $M Q$, where $Q$ is a central projection such that $D(E) \geqq Q / m$, $m$ an integer. The preceding paragraph proves that a maximal orthogonal family of central projections $\left\{Q_{\alpha}\right\}$ such that there exist center states $\left\{\phi_{n}\right\}$ of $M Q_{\alpha}$ satisfying $\phi_{n \alpha}\left(B^{*} B\right) \leqq(n+1) \phi_{n \alpha}\left(B B^{*}\right) / n$, for each $B$ in $M Q_{\alpha}$, has sum $I$. Then $\phi_{n}$ defined on $M$ by: $\phi_{n}(B)$ $=\sum_{\alpha} \phi_{n \alpha}\left(B Q_{\alpha}\right)$, is a center state of $M$ such that $\phi_{n}\left(B^{*} B\right)$ $\leqq(n+1) \phi_{n}\left(B B^{*}\right) / n$, and the proof is complete.

\section{BIBLIOGRAPHY}

1. L. Alaoglu, Weak topologies of normed linear spaces, Ann. of Math. vol. 41 (1940) pp. 252-267.

2. J. Dixmier, Les algèbres d'opérateurs dans l'espace hilbertien, Paris, GauthierVillars, 1957.

3. R. Kadison, On the additivity of the trace in finite factors, Proc. Nat. Acad. Sci. U.S.A. vol. 41 (1955) pp. 385-387. 
4. - Unitary invariants for representations of operator algebras, Ann. of Math. vol. 66 (1957) pp. 304-379.

5. R. Kadison and I. M. Singer, Extensions of pure states, Amer. J. Math. vol. 81 (1959) pp. $383-400$.

Columbia University 